secretory tissue, for the number of alveoli is increased, and the epithelial cells themselves, instead of being cubical, are columnar. It is evident that the thyroid gland in exophthalmic goitre has a strong resemblance to a portion of gland which has undergone compensatory hypertrophy, and which is in a condition of over-activity. We know very little about the nature of the secretion of the thyroid gland in exophthalmic goitre. It is probable that it is altered in quality as well as in quantity. Anyhow, there is a satisfactory anatomical basis for considering the symptoms as due to an over-activity of the gland. Clinical evidence also gives support to this view. Improvement occurs in many cases when as a result of operation the secretory activity of the gland is diminished-i.e., either by excision of part of the gland or by ligature of some of its vessels.

In an article in the Practitioner for November, 1896, Mr. Carless reviews the treatment of exophthalmic goitre. In considering the thyroid theory of the disease he states that the treatment by belladonna succeeds by diminishing the thyroid secretion. Thyroid given in moderate doses has sometimes produced some of the features of exophthalmic goitre, but this may possibly be due to toxic products of putrefaction. If given in exophthalmic goitre it increases for a time the cardiac and nervous symptoms. In discussing the operative treatment of the disease he refers to the symptoms which I have described as follows: "The deathrate amounts to not less than 12 per cent. This is due in a measure to a curious condition which not infrequently superzenes after an apparently clean and successtul operation. All goes well for a few hours and then suddenly the temperature rises to $103^{\circ}$ or $104^{\circ} \mathrm{F}$, the pulse runs up to a high rate, the urine is loaded with albumin, the patient become delirious or comatose, and finally dies perhaps within fifteen hours of the operation." This description coincides in the main features with the description I have given. With regard to the cause Mr. Carless adds that "it has been suggested that it is an excessive secretion of the thyroid secretion brought about by handling the tumour or by a stimulation of the secretive activity by the anæsthesia. I fas occurred after the simplest cases as well as after the more serious ones, but curiously has never been noticed after dealing with simple goitres." The last statement can scarcely hold true any longer, and though I have not found records of published cases of these symptoms occurring after operation on simple goitre I have heard of such cases. The clinical effects of giving an excess of thyroid also support this view. For example, in a case described by Murray a woman took by mistake ninety.two grammes of thyroid gland in eleven days. The symptoms she suffered from were tachycardia, rapid respiration, exophWhalmos, tremor, rise of temperature, and insomnia, symptoms analogous to the condition to which I have drawn attention.

In the two cases above described which showed symptoms cysts were removed from the thyroid gland and a considerable quantity of the fluid escaped into the wound. Hence it would easily be absorbed by the lymphatics, and this, I may say, is the way in which it has been shown that the thyroid secretion normally is conveyed from the gland into the circulation. I therefore submit that the symptoms of exophthalmic goitre, of cases where thyroid has been administered in excess medicinally, and of cases where during operation the contents of a cyst have been emptied into the wound, bear an intimate relation to one another, and that they are due to an excess of thyroid secretion in the circulation. Further, in support of this view, and against the view that the symptoms are due to a want of thyroid, I would point out that in these cases only unhealthy thyroid is removed, that the symptoms are independent of the amount removed or left behind, that they occurred in Case 4, where only a cyst was removed and plenty of thyroid was left, and especially that they occur in exophthalmic cases where only one lobe is removed.

[The writer then referred to Mr. Horsley's explanation of the symptoms in Case 1 as being due to lack of thyroid secretion, to which cause also he attributes the symptoms of exophthalmic goitre. He proceeded to describe fully the symptoms of cachexia strumipriva.]

The subject can only be solved by experimental investigation and by accurate clinical observation coupled with a more complete publication of unsuccessful cases. I submit that none of the important researches which I have quoted offers any fundamental objection to the following proposifions: (1) that the symptoms to which I have been drawing attention-namely, rapid pulse and respiration and restlessness-are analogous to those of exophthalmic goitre; (2) that they are due, as in that complaint, to the absorption by the lymphatics into the circulation of a perverted secretion of the thyroid, not to the absence of normal thyroid; and (3) that in the cases where these symptoms have been most frequently seen after thyroidectomy - namely, after operation for exophthalmic goitre - it is due to squeezing out some secretion while handling the tumour; and that in the cases I have described it entered by the cyst being ruptured into the wound.

[The writer concluded by urging the importance of guarding against these serious symptoms by taking as much care to prevent the contents of a thyroid cyst from entering the wound as a surgeon takes in dealing with a fluid tumour of the abdomen. Should the symptoms arise the hypodermic injection of morphia, he considers, seems to promise the best results ]

Gloucester-terrace, $\mathrm{w}$.

\section{AN UNUSUAL CASE OF WOUND OF THE HEART; RECOVERY.}

By SAMUEL PRIOR, M.B., C.M. Glasg.,

assistant phystclan to the woodILe asYLUM, LENzIE, gLasgow.

WouxDs of the heart are serious both from the immediate danger of wounding the heart itself and from the complications that are apt to arise. Recovery or even partial recovery is all the more satisfactory. The following case, ending in complete recovery, may be interesting.

The patient, a man aged forty-three years, was an epileptic whose mental decadence was not much advanced. The fits were infrequent and usually slight-of the character of petit mal-though he had occasionally well-marked convalsions. He was a confirmed pickpocket and hoarded up pins, nails, pieces of wire, \$c., which were, as a rule, used by patients for cleaning tobacco pipes. In the year 1890 he had shown a strong suicidal tendency by inflicting a deep wound on his neck. He is a type of patient well known in asylums, requiring careful and strict supervision day and night. Last April he made another suicidal attempt. He cunningly chose the time when the patients were getting up and the attendants were very busy for this determined though unsuccessful attempt. The attendant in charge in the sickroom observed the patient doing something unusual, and on removing the bed-clothes was astonished to find an iron pin projecting from the chest in the region of the heart. 'The exact situation was $1 \frac{3}{4}$ inches below and 1 inch internal to the left nipple. This pin was directed slightly upwards and inwards. It moved vigorously with every beat of the heart and travelled about an inch upwards and downwards every time. On examination the pin was felt to be firmly embedded in the wall of the heart, which was felt tugging at the inner end of the pin. Removal of this peculiar foreign body was not undertaken without some misgivings as to the result. The pin was rotated and gently withdrawn; the part within the chest measured 3 inches, making a total length of 4 inches of $\frac{3}{16}$-inch iron wire. The patient was collapsed; be had a small, feeble pulse, 64 per minute ; respiration was shallow, 24 per minute; and the skin was cold. There was practically no hæmorrhage from the wound. No external hæmorrhage appeared subsequently to this, and there were no signs of effusion of blood or serum into the pericardium. The patient kept his eyes closed. He did not resist an examination of the pupils, which were dilated and reacted but slightly to light. When spoken to he would not answer. I thought he was conscious of all that was said, and subsequent events made this almost certain. The wound was dressed antiseptically, and there was no visible suppuration at any time. On the day following the infliction of the wound there was some subcutaneous emphysema round the wound. The physical signs of pneumothorax appeared, but this and the emphysema disappeared again in twenty-four hours. The temperature, pulse, and respiration rates rose to a maximum thirty-six bours after the wound was made. The records were as follows: at 7 P.M. the temperature was $100.8^{\circ} \mathrm{F}$., the pulse was 112 , and the respiration was 36 ; at 9 P.M. the temperature was $101.0^{\circ}$ the pulse was 109 , and the respiration was 32 ; at 11 P.M. the 
temperature was $101 \cdot 0^{\circ}$, the pulse was 105 , and the respiration was 38. The temperature remained slightly febrile for a week and then became normal. The patient would not take food or drink for four days, and seemed indifferent to everything about him. On the fourth night he asked for a drink of water. The following day he looked round the room several times when he thought no one was watching him. He asked why he was kept in bed and wished to get up. Several pieces of wire were found in his bed, showing that he was still occupied with suicidal projects. He was removed to another bed, it being supposed, and rightly so, that he had concealed these in the wire mattress. A handful of pieces of wire, needles, pins, \&c., was taken out of the mattress. He was excessively annoyed at having his bed changed and the wire, \&c., removed. He told one of the attendants he "hadn't succeeded yet, but would try again and succeed." His appetite soon returned and he recovered his usual health. Namerous pulse tracings were taken. Soon after the pin was removed the tracing showed a low tension pulse with almost full dicrotism and slight irregularity. On the third day the tracing showed a better tension pulse; the dicrotism was nearly gone. On the fourth day there was still some dicrotism; the tracings showed great amplitude of the percussion wave. Normal tracings were obtained on the succeeding days. There are now (nearly six months after) no signs of pericardial adhesion. The only evidence left of the injury is a reddish linear scar half an inch long, directed downwards and outwards.

It is rather surprising that such a thick and dirty piece of wire prodaced no suppuration or irritation of the pericardium. The pin must have entered at least two inches into the wall of the left ventricle, but did not enter the cavity of that ventricle. The patient seemed rather surprised and ashamed at his failure. Althongh kept under the closest supervision day and night he declares his intention plainly of "trying to manage it yet." Life for him has no attraction-it is indeed a weariness to the flesh; he has reached a point where "the terrors of life outweigh the terrors of death."

Glasgow.

\section{ON THE SURGICAL TREATMENT OF SPASTIC PARALYSIS IN CHILDREN.}

By J. JAOKSON CLARKE, M.B. LOND., F.R.C.S. ENG., ASSISTANT SURGEON-AT THE NORTH-WEST LONDON AND CITY ORTHOP EDIC HOSPITALS.

WherRever many disabled children are seen a certain proportion of them will be found to be suffering from spastic paralysis. Though recognised from the earliest times the affection was first systematically described by the English orthopædic surgeon, Little, who rightly ascribed the majority of cases to superficial lesions of the cerebral convolutions resulting from meningeal bæmorrhage indused by the pressure of the maternal pelvic bones on the foetal skull. It is naturally commonest in first.born children and is usually congenital. Some cases, however, are not congenital, but arise in infancy from thrombosis of the superficial cerebral vessels during the course of fevers, such as measles, scarlet fever, and influenza. All grades of the affection are met with. There may be muscular rigidity of the neck and trunk as well as of the limbs, or only the lower limbs may be affected. The intellectual functions may be totally destroyed or completely intact. In the latter case it has been supposed that the affection is of spinal origin, though there is no satisfactory proof that this invariably holds good. As an introduction to the surgical aspects of spastic paralysis one of these slighter cases may be briefly considered, a case in which the intellect is unaffected and the spasm is limited to the muscles of the leg with resulting talipes equinus, equinovarus, or equino-valgus. Electricity and massage have been tried till the parents of the child have grown weary and the uselessness of these measures has become obvious. Recourse has then been had to retentive apparatus in order to try to effect a gradual correction of the deforwity. Hope is awakened, and with patient endeavour the apparatus is given every chance of effecting its purpose. But after months or years of perseverance the uselessness of retentive apparatus alone becomes evident. It is uncomfortable ard wearisome to the little patient, increasing the spasm of the stronger muscles, and in this way rather impeding than belping progression. And whenever the appa. ratus is left off for a moment the deformity at once returns with, if anything, greater rigidity than ever. At last, with reluctance and sometimes with bot little confidence, operative treatment is suggested, and the parents of the child accept it as a last desperate chance. If the operation is done thoroughly, every resisting tendon being completely severed and the limb fixed at once in an over-corrected position and so left for four or five weeks, it is found that at the end of this time the tendency to return to the deformity is greatly diminished and walking is again possible to the patient, and, if the latter is intelligent, by exercise of the will the tendency to deformity may soon be entirely overcome, so that retentive apparatus may be dispensed with in the day and at night only a light and simple shoe may be required to render the cure a permanent one.

If a case of infantile paralysis is compared with one of spastic paralysis, a marked contrast may be observed. Infantile paralysis is caused by destruction of the cells of the anterior cornua of the spinal cord; spastic paralysis is attributable to partial disablement of the cells of the motor cortex or to partial blocking of the pyramidal tracts. In infantile paralysis massage and Faradaism in the early stages help the recovery of any musculas fibres of which the nutritive nerve cells have not been completely destroyed; in spastic paraplegia massage is of no nse apart from complete tenotomy. In infantile paralysis suitable apparatus prevents the occurrence of deformity; in spastic paralysis the deformity is only over. come by the use of much force and at the cost of some distress to the patient. In deformity due to infantile paralysis tenotomy, \&c., are only employed to bring the limbs into workable shape and, save in the very slightese cases, the retentive apparatus must be worn for the rest of the patient's life; in spastic paralysis efficient tenotomy, by weakening the muscles the persistent over-action of which produces the deformity, tends to restore the balance of muscular power, and in most cases this may be so faw successful that retentive apparatus can be dispensed with.

Lorenz, ${ }^{1}$ who has recently published an important series of observations on this topic, attributes the good effect of thorough tenotomy in spastic cases, to the shortening of the bellies of the tenotomised muscles, so that their range of action is diminished. In severe cases of spastic contraction of the knee Lorenz recommends excision of from one inch to one and a half inch of the tendons of the biceps, semi-tendinosus, and semi-membranosus muscles and even after such tenectomies and subsequent straightening of the knee Lorenz has never observed a failure of union in the widely-separated ends of the tendons. Thus, after an ordinary tenotomy failure of union need not be feared. For the marked adductor spasm, which, by causing the knees to press against each other, is often such a serious hindrance to progression, Lorenz has found that in mild cases forcible stretching of the adductors combined with manipulation is successful, in more severe cases subcutaneous tenotomy answers, whilst in the most pronounced cases he has successfully $€ x$ xised a secticn of the obturator nerve - an operation of some difficulty in the adducted position of the limb.

Indications for operative treatment.-Before deciding to undertake the operative treatment of any case various factors have to be weighed. In the first place, the condition of the intellectual faculties must be considered. Often the mind is altogether vacant and in such cases it is not worth while to rectify malpositions. Sometimes the condition is complicated by bilateral athetosis (general chorfa) and this may be taken as a distinct contra-indication. Epilepsy is a somewhat rare complication and would also be an argument for non.interference. General rigidity renders improvement of the condition of the legs of but little service to the patient. In diagnosis the cases of infantile cerebral degeneration with symmetrical changes at the macula, first observed by Waren Tay and recently described by Kingdon and Risien Russell, ${ }^{2}$ must be borne in mind. Such cases begin in infancy and end fatally about the end of the second year. They are marked by progressive paralysis and some rigidity.

The paraplegic forms of spastic paralysis are the more

A. Lorenz: Wiener Klinische Rundschau, Nos. 21-26, 1897. 2 Transactions of the Royal Medical and Chirurgical Society; 1897 